Research Article

\title{
Bayesian Estimation of Ammunition Demand Based on Multinomial Distribution
}

\author{
Kang Li $\left(\mathbb{D}\right.$, Xian-ming Shi $\mathbb{D}^{D}$, Juan Li, Mei Zhao, and Chunhua Zeng \\ Shijiazhuang Campus, Army Engineering University, Shijiazhuang 050003, China \\ Correspondence should be addressed to Xian-ming Shi; sxm@nudt.edu.cn
}

Received 20 February 2021; Revised 31 March 2021; Accepted 22 April 2021; Published 29 April 2021

Academic Editor: Ya Jia

Copyright (c 2021 Kang Li et al. This is an open access article distributed under the Creative Commons Attribution License, which permits unrestricted use, distribution, and reproduction in any medium, provided the original work is properly cited.

\begin{abstract}
In view of the small sample size of combat ammunition trial data and the difficulty of forecasting the demand for combat ammunition, a Bayesian inference method based on multinomial distribution is proposed. Firstly, considering the different damage grades of ammunition hitting targets, the damage results are approximated as multinomial distribution, and a Bayesian inference model of ammunition demand based on multinomial distribution is established, which provides a theoretical basis for forecasting the ammunition demand of multigrade damage under the condition of small samples. Secondly, the conjugate Dirichlet distribution of multinomial distribution is selected as a prior distribution, and Dempster-Shafer evidence theory (D-S theory) is introduced to fuse multisource previous information. Bayesian inference is made through the Markov chain Monte Carlo method based on Gibbs sampling, and ammunition demand at different damage grades is obtained by referring to cumulative damage probability. The study result shows that the Bayesian inference method based on multinomial distribution is highly maneuverable and can be used to predict ammunition demand of different damage grades under the condition of small samples.
\end{abstract}

\section{Introduction}

Ammunition supply support is a key element in the formation of combat effectiveness [1]. In the information war, ammunition supply support plays an increasingly prominent role, and ammunition demand forecasting, as a basic work, becomes increasingly concerned [2]. Domestic relevant scholars have made systematic research on ammunition demand forecasting methods.

Traditionally, there are three ammunition demand prediction methods: ammunition consumption standard correction method, empirical deduction algorithm, and theoretical calculation method. The ammunition consumption standard correction method is based on the ammunition consumption standard, which is modified according to actual combat conditions to predict the ammunition demand, but with the rapid development of weapons and ammunition, the formulation of ammunition consumption standards cannot be followed up in time. In an empirical deduction algorithm, the law of ammunition consumption is summarized by analyzing typical war cases, and ammunition demand is predicted according to the future war development trend and ammunition consumption characteristics. Considering historical limitations in respect to the complexities of the future combat mode and the diversity of the combat means, the empirical deduction algorithm is difficult to apply. The theoretical calculation method is a mathematical method for calculating ammunition consumption based on military indicators, such as the number of weapons and equipment. It can be further classified into the task quantity method and the battle scenario method. When the theoretical calculation method is used for ammunition demand estimation, large amounts of accurate and reliable trial data are required to be as a basis [3].

With the development of ammunition demand prediction theory, the simulation prediction method based on military operations, the prediction method based on an intelligent algorithm, and the combination forecasting method [4, 5] are gradually adopted by researchers. The simulation prediction method based on military operations refers to the military operations methodologies such as the 
Lanchester equation and combat simulation in ammunition demand prediction. Xiao et al. [6] introduced the matrix of fire distribution coefficient, improved the Lanchester combat equation, and established an air-to-air missile consumption prediction model as well as an air combat model adapted to modern warfare. The prediction method based on intelligent algorithms refers to the application of smart algorithms such as artificial neural networks, support vector machines, particle swarm optimization, and Bayesian algorithm in ammunition demand prediction. Tian et al. [7] have incorporated variant particle swarm optimization into deep neural networks to make aviation ammunition training consumption prediction. In the combination forecasting method, the strengths of every single prediction model can be put to good use, yet a large amount of ammunition consumption data is needed for [8].

In order to solve the problem of a small amount of test data caused by economic problems in ammunition demand prediction, the Bayesian method is included in the research scope [9, 10]. Zhang et al. [11] studied the problem of uncertainty quantification and propagation when data for characterizing probability distributions are scarce. Matthias et al. considered the case where only a very scarce set of experimentally obtained eigenfrequencies and eigenmodes are available and proposed an improved approach for the quantification of multivariate interval uncertainty for highdimensional models under scarce data availability [12, 13]. Zhao et al. [2] proposed a Bayesian inference method for ammunition demand based on Gompertz distribution and solved the problem of ammunition demand prediction in the case of insufficient samples.

The prior information comes from expert information and field test information in the process of using the Bayes method. To make better use of the prior information, it is necessary to fuse them. The D-S theory is widely used in multisource information fusion because of its effectiveness in data fusion. Tang et al. [14, 15] generated generalized basic probability assignment (GBPA) based on the triangular fuzzy number model under the open world assumption. Zhang et al. [16] focused on the determination of Basic Probability Assignment (BPA) and proposed a BPA determination method based on triangular fuzzy members under the assumption of an open world. Experts' understanding of ammunition effectiveness is based on ammunition effectiveness test data, and the field trial information is ammunition effectiveness data obtained from the field trial. There is no potential conflict between these two pieces of evidence. Therefore, the Dempster combination rule is used to fuse expert information and the field trial in this paper.

Ammunition demand refers to the amount of ammunition needed for a target to reach a certain degree of damage. Without considering the cumulative effect of ammunition damage, the calculation of ammunition demand comes from the single-shot damage probability of ammunition. In fact, the probability that the target reaches a certain degree of damage is stable in the process of ammunition damage. Therefore, this paper assumes that the damage result of the target obeys multinomial distribution. Obtaining multinomial distribution parameters becomes a key link, and its value depends on expert experience and field test data.

Based on the above analysis, a Bayesian inference method of ammunition demand based on multinomial distribution is proposed. It considers the different damage grades in combat ammunition hitting and the actual demand of ammunition prediction under the condition of a small sample size. The multinomial distribution is used for describing the damage result of the target and the D-S theory is used for fusing expert information and field test information. And the ammunition demand as reaching different damage grades is forecasted based on Bayesian statistics, which provide a method for solving the ammunition demand prediction under the condition of multiple damage grades and small samples.

\section{Bayesian Inference Method for Ammunition Demand}

2.1. Prior Distribution Representation of Previous Information. Compared with the classical statistical inference method, the Bayesian inference method integrates general information, sample information, and prior information and regards the parameters to be estimated as random variables. Assuming that the overall distribution is $p(x \mid \theta)$, the posterior distribution of parameter $\theta$ can be obtained by the Bayesian formula:

$$
P(\theta \mid x)=\frac{P(x \mid \theta) \pi(\theta)}{m(x)},
$$

where $m(x)$ is the edge probability corresponding to ammunition consumption data and independent of the parameter $\theta, \theta$ is an unknown parameter of the overall distribution, and $x$ represents sample data; then, the Bayesian formula can be rewritten as

$$
P(\theta \mid x) \propto P(x \mid \theta) \pi(\theta) .
$$

It can be seen from formula (2) that prior distribution, as is crucial for the calculation of posterior distribution function, is determined with the need for scientific and reasonable previous information. Even under the condition of small samples, a certain amount of prior information is needed. For ammunition damage data collection, the prior information stems from expert experience and field trial; that is, the ammunition required to achieve different damage grades is

$$
\begin{aligned}
\varphi^{\Gamma_{1}} & =\left\{\varphi_{1}^{\Gamma_{1}}, \varphi_{2}^{\Gamma_{1}}, \ldots\right. \\
\varphi^{\Gamma_{2}} & =\left\{\varphi_{1}^{\Gamma_{2}}, \varphi_{2}^{\Gamma_{2}}, \ldots\right. \\
\ldots & \\
\varphi^{\Gamma_{n}} & =\left\{\varphi_{1}^{\Gamma_{n}}, \varphi_{2}^{\Gamma_{n}}, \ldots\right.
\end{aligned}
$$

among which $\varphi_{r}^{\Gamma_{i}}(i=1,2, \ldots, n)$ represents the ammunition demand.

The methods to determine the prior distribution function include the maximum entropy method commonly used in engineering, the conjugate distribution method with 
known overall distribution, and the expert scoring method. Assuming that the conditions of each shooting test are basically the same and each shooting is independent, each shooting test has $r(r>2)$ different damage levels, but only one of them will appear in the result. In each shooting test, each result has its own occurrence probability, and the sum of the occurrence probabilities of all results is $100 \%$, so the target damage result can be described as multi-item distribution.

To facilitate the calculation and reasonably explain the parameters, the conjugate distribution method is used to determine prior distribution in this paper. Given that the conjugate prior distribution of multinomial distribution is Dirichlet distribution, the previous probability density function of ammunition demand $\varphi^{\Gamma_{i}}(i=1,2, \ldots, n)$ is

$$
f\left(\varphi_{1}, \ldots, \varphi_{n} ; \alpha_{1}, \ldots, \alpha_{L}\right)=\frac{1}{B(\alpha)} \prod_{v=1}^{L}\left(\varphi_{i}\right)^{\alpha_{v}-1}
$$

where $B(\alpha)$ is a multivariate beta function; that is,

$$
\begin{aligned}
B(\alpha) & =\frac{\prod_{i=1}^{L} \Gamma\left(\alpha_{i}\right)}{\Gamma\left(\alpha_{0}\right)}, \\
\alpha_{0} & =\sum_{i=1}^{L} \alpha_{i},
\end{aligned}
$$

where $\Gamma(\alpha)$ is a Gamma function. $L \geq 3$ represents the hyperparameter of Dirichlet distribution.

The prior distribution function of ammunition demand $\varphi^{\Gamma_{i}}(i=1,2, \ldots, n)$ can be obtained as follows:

$$
F(\varphi, \alpha, c)=\int_{\Omega} f\left(\varphi_{1}, \ldots, \varphi_{n} ; \alpha_{1}, \ldots, \alpha_{K}\right) \mathrm{d} x
$$

where $\Omega=R_{\varphi} \cap[0, b], \varphi \in R_{\varphi}, 0<b \leq 1$.

To obtain more accurate Bayesian inference results, the fusion of different prior information should be attended to in the application of Bayesian methods. Expert information comes from the damage data of different similar targets, which has a certain credibility. The field test data may also have certain deviations because of the small number of samples. The D-S theory can effectively fuse and correct the deviation. Therefore, the D-S theory is introduced in this paper for the fusion of prior information.

2.2. Multisource Prior Information Fusion Based on D-S Theory. Before the firing trial, experts could have a specific prior understanding of the ammunition demand required to reach the damage grade. To utilize the information and the field trial data simultaneously, multisource Bayes prior information is fused based on the D-S theory, and the fusion formula is $[17,18]$

$$
m_{1 \oplus 2}(A)= \begin{cases}\frac{\sum_{B \cap C=A} m_{1}(B) m_{2}(C)}{1-K}, & A \neq \varnothing, \\ 0, & A=\varnothing,\end{cases}
$$

in which $K=\sum_{B \cap C=\varnothing} m_{1}(B) m_{2}(C), A$ indicates different damage levels, and $m_{1}(B)$ and $m_{2}(C)$ represent mass functions, which satisfy

$$
\begin{aligned}
m(\varnothing) & =0, \\
\sum_{A \subseteq \Theta} m(A) & =1 .
\end{aligned}
$$

In this paper, the D-S theory is used to fuse expert information and field test data, as shown in Table 1.

According to the data in Table 1,

$$
1-K=\sum_{i=1}^{N+1} m_{1}\left(A_{i}\right) m_{2}\left(A_{i}\right)
$$

After fusing expert information and field test information, the prior information of damage grade is as follows:

$$
\begin{aligned}
m\left(A_{i}\right) & =m_{1}\left(A_{i}\right) \oplus m_{2}\left(A_{i}\right) \\
& =\frac{m_{1}\left(A_{i}\right) m_{2}\left(A_{i}\right)}{1-K} \\
& =\frac{m_{1}\left(A_{i}\right) m_{2}\left(A_{i}\right)}{\sum_{i=1}^{N+1} m_{1}\left(A_{i}\right) m_{2}\left(A_{i}\right)} .
\end{aligned}
$$

According to the analysis of the experimental data of ammunition effectiveness, the identification framework $\Theta$ represents the set of different damage levels. By multiplying the probability value obtained by formula (10) with the number of tests, the target frequency reaching different damage levels can be obtained.

\section{Bayes Inference Model of Ammunition Demand Based on the Multinomial Distribution}

3.1. Multinomial Distribution Hypothesis. Assume that the number of damage grades is $r$ and each firing trial is under nearly the same conditions. $N$ shooting trials are carried out in advance and each shooting produces $r$ basic events. $S_{1}, S_{2}, \ldots, S_{r}$ represent different damage grades. Denote $p_{i}=P\left\{S_{i}\right\}, i=1, \ldots, r$ as the occurrence probability of different damage grades and mark $X_{i}(i=1, \ldots, r)$ to indicate the number of times $S_{i}$ occurred in $N$ strike trials. It can be seen that $\left(X_{1}, X_{2}, \ldots, X_{r}\right)$ obeys the multinomial distribution

$$
\begin{gathered}
P\left(X_{1}=k_{1}, \ldots, X_{r}=k_{r}\right) \\
=\frac{n !}{k_{1} ! \ldots k_{r} !} p_{1}^{k_{1}} \ldots p_{r}^{k_{r}},
\end{gathered}
$$

and satisfies $\sum_{i=1}^{r} k_{i}=N, \sum_{i=1}^{r} p_{i}=1$.

3.2. Posterior Distribution of Dirichlet Conjugate Prior. Zhang et al. [19] studied the effect of prior on prediction and estimation in Bayesian inference. They think that model parameters are shown to have a significant impact on quantified uncertainties. Shitsi et al. [20] think that Jeffreys 
TABLE 1: Basic probability assignment of expert information and field test information.

\begin{tabular}{lcc}
\hline Recognition frameworks & Expert information $m_{1}$ & Field test information $m_{2}$ \\
\hline$A_{0}$ & $m_{1}\left(A_{0}\right)$ & $m_{2}\left(A_{0}\right)$ \\
$A_{1}$ & $m_{1}\left(A_{1}\right)$ & $m_{2}\left(A_{1}\right)$ \\
$\vdots$ & $\vdots$ & $\vdots$ \\
$A_{N+1}$ & $m_{1}\left(A_{N+1}\right)$ & $m_{2}\left(A_{N+1}\right)$ \\
\hline
\end{tabular}

prior is preferred over Uniform Dirichlet prior for Bayesian analysis because it yields longer confidence intervals (or shorter uncertainty interval) at $90 \%$ confidence level. Raiffa and Schlaifer suggested that a conjugate distribution is appropriate for a prior distribution [21], so Conjugate prior distribution is adopted in this paper. The multinomial distribution is used as the likelihood function of ammunition demand data of weapon damage degree. To estimate the ammunition demand, the Dirichlet distribution is the conjugate distribution family selected as the prior distribution to obtain the posterior distribution function of ammunition demand at each damage grade. Assuming that the strike trial is carried out from time $t_{0}$ to time $t_{1}$, the observed result is $o_{1}=\left[k_{1}, k_{2}, \ldots, k_{r}\right]$. The observed result from time $t_{1}$ to time $t_{2}$ is $o_{2}=\left[k_{1}^{\prime}, k_{2}^{\prime}, \ldots, k_{r}^{\prime}\right]$. $o_{1}$ is selected as the prior probability and $o_{2}$ as the conditional probability. According to Bayes theorem, the posterior probability of parameter $p$ can be obtained and expressed as $P\left(p \mid o_{2}\right)$. Both $o_{1}$ and $o_{2}$ obey multinomial distribution, and the multinomial distribution likelihood function followed by $o_{2}$ can be expressed as

$$
P\left(o_{2} \mid p\right)=\operatorname{Mult}(\vec{n} \mid \vec{k}, N)=\left(\begin{array}{c}
N \\
\vec{n}
\end{array}\right) \prod_{i=1}^{r} p_{i}^{k_{i}^{\prime}}
$$

Based on the Bayesian theorem, the posterior distribution density function of the parameter $p$ can be obtained as follows:

$$
\begin{aligned}
f\left(p \mid o_{2}\right) & \\
& =\frac{P\left(o_{2} \mid p\right) f(p)}{P\left(o_{2}\right)} \\
& =\frac{P\left(o_{2} \mid p\right) f(p)}{\int P\left(o_{2} \mid p\right) f(p) \mathrm{d} p} .
\end{aligned}
$$

Let $f(p)=f\left(p \mid o_{1}\right)$, and the posterior distribution density function can be expressed as

$$
\begin{aligned}
& f\left(p \mid o_{2}\right) \\
& =\frac{P\left(o_{2} \mid p\right) f(p)}{\int P\left(o_{2} \mid p\right) f(p) \mathrm{d} p} \\
& =\frac{\prod_{i=1}^{r} p_{i}^{k_{i}^{\prime}}\left(\prod_{i=1}^{r} \Gamma(k+r) / \prod_{i=1}^{r} \Gamma\left(k_{i}+1\right)\right) \prod_{i=1}^{r} p_{i}^{k_{i}}}{\int \prod_{i=1}^{r} p_{i}^{k_{i}^{\prime}}\left(\prod_{i=1}^{r} \Gamma(k+r) / \prod_{i=1}^{r} \Gamma\left(k_{i}+1\right)\right) \prod_{i=1}^{r} p_{i}^{k_{i}} \mathrm{~d} p} \\
& =\frac{\prod_{i=1}^{r} p_{i}^{k_{i}^{\prime}+k_{i}}}{\int \prod_{i=1}^{r} p_{i}^{k_{i}^{\prime}+k_{i}} \mathrm{~d} p} \\
& =\frac{\Gamma\left(k_{i}^{\prime}+k+r\right)}{\prod_{i=1}^{r} \Gamma\left(k_{i}^{\prime}+k+r\right)} \prod_{i=1}^{r} p_{i}^{k_{i}^{\prime}+k_{i}} \\
& =\frac{\Gamma\left(\alpha^{\prime}+\alpha\right)}{\prod_{i=1}^{r} \Gamma\left(\alpha^{\prime}+\alpha\right)} \prod_{i=1}^{r} p_{i}^{\alpha_{i}^{\prime}+\alpha_{i}-1} .
\end{aligned}
$$

It can be seen that the posterior distribution density function obeys Dirichlet distribution.

3.3. Bayesian Analysis of Ammunition Demand Based on the Multinomial Distribution. After using the Dirichlet distribution of conjugate prior distribution of multinomial distribution to obtain the estimated value of posterior distribution parameters, the updated ammunition demand $\varphi^{\Gamma_{i \prime}}(i=1,2, \ldots, n)$ can be obtained by cumulative calculation of ammunition demand reaching different damage grades.

Combining the fused prior information in the light of D-S theory with the Bayesian theorem, the formula of fusion density can be obtained as follows:

$$
f\left(p \mid o_{2}\right)=\frac{P\left(o_{2} \mid p\right) f(p)}{P(A)} .
$$


The fusion posterior density is

$$
f\left(p \mid o_{2}\right)=\frac{\left(\begin{array}{c}
N \\
\vec{n}
\end{array}\right) \prod_{i=1}^{r} p_{i}^{k_{i}^{\prime}}}{\left(\prod_{i=1}^{n} \int \pi_{i}(\theta) \mathrm{d} \theta / \prod_{i=1}^{n} \iint_{\Theta} \pi_{i}(\theta) \mathrm{d} \theta\right)} .
$$

According to formula (15), the posterior estimation of the parameter $p$ can be obtained as follows:

$$
E\left(p_{i} \mid o_{2}\right)=\int_{0}^{1} t f\left(p \mid o_{2}\right) \mathrm{d} t .
$$

The parameter means $\theta_{1}, \theta_{2}, \ldots, \theta_{r}$ corresponding to different damage grades can be obtained after calculation. When the ammunition demand for different damage grades is expected, the cumulative calculation is needed. For example, when moderate damage is to be estimated, severe damage and scrap should be taken into account.

After $\theta_{1}, \theta_{2}, \ldots, \theta_{r}$ is obtained, the probability value $P\left(\theta_{1}\right), P\left(\theta_{2}\right), \ldots, P\left(\theta_{r}\right)$ corresponding to different damage grades can be obtained by cumulative calculation, and the calculation formula is

$$
P\left(\theta_{i}\right)=\theta_{i}+\theta_{i+1} \cdots+\theta_{r}
$$

where $1 \leq i \leq r$.

Equation (17) represents the probability value corresponding to each damage grade during a single strike. When multiple strikes are carried out, it is expressed as follows:

$$
P\left(\varphi^{\Gamma_{i}}, \theta_{i}\right)=1-\left(1-P\left(\theta_{i}\right)\right)^{\varphi^{\Gamma_{i}}} .
$$

The damage of ammunition to the target can be regarded as a random event. When calculating the ammunition consumption, it is necessary to determine the probability critical value $\Lambda$ when the ammunition consumption is up to a certain level and the damage reaches a specific level. It is considered that when the cumulative damage probability exceeds the damage probability significant value $\Lambda$, the target will be successfully damaged. Assuming that $\varphi^{\Gamma_{r}^{*}}$ is the ammunition demand when reaching damage grade $r$, the discriminant of ammunition demand is

$$
\sum_{\varphi^{\Gamma_{i}=0}}^{\varphi^{\Gamma_{n}^{*}}-1} P\left(\varphi^{\Gamma_{i}}, \theta_{i}\right) \leq \Lambda \leq \sum_{\varphi^{\Gamma_{i}}=0}^{\varphi^{\Gamma_{n}^{*}}} P\left(\varphi^{\Gamma_{i}}, \theta_{i}\right)
$$

According to the above formula, the best ammunition demand $\varphi^{\Gamma_{r}^{*}}$ for $r$ damage grades can be determined.

\section{Model Solving}

4.1. Compatibility Test of Prior Information. The premise of Bayes statistical inference method using prior information is that previous information can reflect the statistical characteristics of parameters; that is, advance information and outfield trial data approximately obey the same distribution [22]. In this research, both the prior information and shooting range trial data follow the multinomial distribution. To judge whether they meet the consistency condition, it is necessary to check the compatibility of prior information.

Compatibility test consists of two methods: a parametric test and a nonparametric test [23]. Because the variance of the prior information population is unknown, the nonparametric test method does not involve the parameters of the population distribution in the inference process, so the nonparametric test method is used for data tests in this paper.

It is assumed that the observed values of samples from populations $X$ and $X^{\prime}$ are $\left(x_{1}, x_{2}, \ldots, x_{u}\right)$ and $\left(x_{1}^{\prime}, x_{2}^{\prime}, \ldots, x_{w}^{\prime}\right)$, respectively, where $w>u>1$. The test hypothesis is that $Y: X$ and $X^{\prime}$ are in the same population. Mix the two groups of samples and arrange them from big to small; the order statistics can be obtained [24]:

$$
z_{1} \leq z_{2} \leq \cdots \leq z_{u+w}
$$

If $x_{k}=z_{j}$, consider that the rank $r_{k}$ in the mixed sample satisfies $r_{k}(x)=j$. If some of the observed values are the same, take the rank as the average of the subscripts sum in mixed samples, and define the rank of sample $\left(x_{1}, x_{2}, \ldots, x_{u}\right)$ in the mixed sample $z_{1} \leq z_{2} \leq \cdots \leq z_{u+w}$ as follows:

$$
T=\sum_{k=1}^{u} r_{k}(x) .
$$

In the formula, $T$ is the statistical test value. When $T$ is neither too large nor too small, the two groups of samples meet the condition of consistency. Given the significance level $\alpha$ and the acceptance interval of the inspection amount $\left(T_{a}, T_{b}\right)$, the inspection criteria are

$$
P\left(T_{a}<T<T_{b} \mid Y\right)=1-\alpha .
$$

When $T$ meets the above conditions, the two groups of samples are compatible, otherwise incompatible.

Up to this point, the compatibility test of the prior information of ammunition demand estimation is completed, and further solutions can be made.

4.2. Basic Principle of the Markov Chain Monte Carlo Method. According to the fusion posterior distribution density function (15), to get posterior integral and posterior estimation values, complex high-dimensional calculus needs to be calculated, which is difficult to realize. In this paper, Markov chain Monte Carlo (MCMC) is adopted to realize the numerical calculation of high-dimensional integral. Its basic principle is as follows:

(a) Constructing Markov chain: start from the initial state $h_{0}$ and make state transition on the Markov chain. According to the Markov chain convergence theorem, probability distribution $h_{i}(x)$ will converge to stationary distribution $h(x)$.

(b) Generating samples: starting from a particular point $X^{(0)}$ in $\phi$, use the Markov chain constructed in (a) for sampling simulation, and create a sequence of points $X^{(1)}, X^{(2)}, \ldots, X^{(g)}$. 
(c) Monte Carlo integration: the marginal distribution of each state cannot be considered as a stable distribution after $q$ iterations before the convergence of the Markov chain, so it is necessary to eliminate the first $q$ iterations and use the following $g-q$ iterations to calculate. Then, the expected estimate value of any function $f(X)$ can be obtained $[25,26]$; that is, the integral value is

$$
E[f(X)]=\frac{1}{g-q} \sum_{t=q+1}^{g} f\left(X^{(t)}\right) .
$$

4.3. MCMC Solution Flow Based on Gibbs Sampling. The Metropolis-Hastings method and the Gibbs sampling method are two widely used MCMC methods in Bayesian analysis. The Markov chains constructed by which are reversible [27]. The Gibbs sampling method proposed by Geman has the advantage of dealing with high-dimensional features and is used to simplify the complex problem of multiple related parameters into a simple one in which only one parameter needs to be dealt with at a time. [28]. In this paper, the MCMC method based on Gibbs sampling is used to solve the posterior estimation [29-31]. The Specific steps are as follows:

Step 1: determine the posterior conditional distribution of parameters. Generating simulation sample data from posterior conditional distributions is easier than from parameter vectors. [32]. Therefore, the posterior conditional distribution of parameters is determined according to formula (24) as follows:

$$
\left\{\begin{array}{l}
h_{1}\left(\theta_{1} \mid \theta_{2}, \ldots, \theta_{m}, X\right) \\
h_{2}\left(\theta_{2} \mid \theta_{1}, \ldots, \theta_{m}, X\right) \\
\vdots \\
h_{m}\left(\theta_{m} \mid \theta_{1}, \ldots, \theta_{m-1}, X\right) .
\end{array}\right.
$$

Step 2: determine the initial value, select an initial state point $\theta^{(0)}=\left(\theta_{1}^{(0)}, \theta_{2}^{(0)}, \ldots, \theta_{m}^{(0)}\right)$, and set the iteration times as $M$.

Step 3: sampling iteration. Extract sample values of parameters $\theta^{(j)}$ from posterior conditional distribution in the following way:

$\theta_{1}^{(1)}$ is extracted from the posterior conditional distribution $h_{1}\left(\theta_{1} \mid \theta_{2}^{(0)}, \ldots, \theta_{m}^{(0)}, X\right)$

$\theta_{2}^{(1)}$ is extracted from the posterior conditional distribution $h_{2}\left(\theta_{2} \mid \theta_{1}^{(1)}, \ldots, \theta_{m}^{(0)}, X\right)$

$\ddot{\theta}_{m}^{(1)}$ is extracted from the posterior conditional distribution $h_{m}\left(\theta_{m} \mid \theta_{1}^{(1)}, \ldots, \theta_{m-1}^{(1)}, X\right)$

A new sample is $\left(\theta_{1}^{(1)}, \theta_{2}^{(1)}, \ldots, \theta_{m}^{(1)}\right)$

Step 4: repeat the iteration of Step 3 and stop when the maximum iteration number $M$ is reached, so the simulation samples of posterior distribution parameters are obtained.
Step 5: calculate the parameters. In this paper, sample mean serves as the parameter point estimation value in this paper:

$$
\begin{aligned}
& \widehat{\theta}_{1}=\frac{\theta_{1}^{(1)}+\theta_{1}^{(2)}+\cdots+\theta_{1}^{(M)}}{M} \\
& \widehat{\theta}_{2}=\frac{\theta_{2}^{(1)}+\theta_{2}^{(2)}+\cdots+\theta_{2}^{(M)}}{M} \\
& \vdots \\
& \hat{\theta}_{m}=\frac{\theta_{m}^{(1)}+\theta_{m}^{(2)}+\cdots+\theta_{m}^{(M)}}{M} .
\end{aligned}
$$

So far, the estimated values of parameters corresponding to each damage grade are obtained, which can be used for Bayes inference of ammunition demand.

\section{Example Analysis}

It is assumed that five damage grades are caused after ammunition hitting, and the conditions of each shooting trial are nearly the same. Sixty shooting trials are conducted in advance and five essential events occurred in each strike, corresponding to five different damage grades, namely, zero damage, mild damage, moderate damage, severe damage, and scrapping. To test the technical performance of hightech weapons and ammunition, an individual unit has carried out a specific actual installation and firing trial in the shooting range, out of which a certain kind of ammunition of high-tech weapons is selected as the research object in this study. Given the low frequency of firing and the indefinite amount of ammunition needed to reach the corresponding damage grade, in this study, the Bayesian statistical inference method is used to predict the amount of ammunition required to reach different damage grades while applying a small sample statistical analysis method to data analysis.

Combining expert information with field trial data, the frequency distribution for reaching different damage grades under the condition of striking the same target is shown in Table 2.

Python software is used to count the number of trials, and visual processing is shown in Figure 1. It can be seen that it is reasonable to assume the strike situation with multinomial distributions.

In this paper, through the Markov chain Monte Carlo method, the statistical inference in the WinBUGS software environment is made. With the Gibbs sampling algorithm, three Markov chains are generated by sampling from the complete conditional probability distribution. To estimate the posterior for the parameters $\theta_{1}, \theta_{2}, \theta_{3}, \theta_{4}, \theta_{5}$ of the multinomial distribution corresponding to different damage grades, multinomial distribution is selected as a likelihood function with reference to formula (11), and Dirichlet distribution is regarded as a conjugate prior distribution with the application of equation (4). The posterior density estimation of parameters can be obtained by setting the number 
TABLE 2: Target frequency distribution for reaching different damage grades.

\begin{tabular}{lcccccc}
\hline Serial number & Number of trials & Zero damage & Mild damage & Moderate damage & Severe damage & Scrapping \\
\hline 1 & 60 & 5 & 10 & 12 & 18 \\
2 & 60 & 3 & 8 & 12 & 15 \\
3 & 60 & 7 & 8 & 15 & 14 \\
4 & 60 & 8 & 12 & 13 & 14 \\
5 & 60 & 6 & 12 & 10 & 12 \\
6 & 60 & 15 & 12 & 12 & 12 \\
7 & 60 & & 12 & 13 & 10 \\
\hline
\end{tabular}

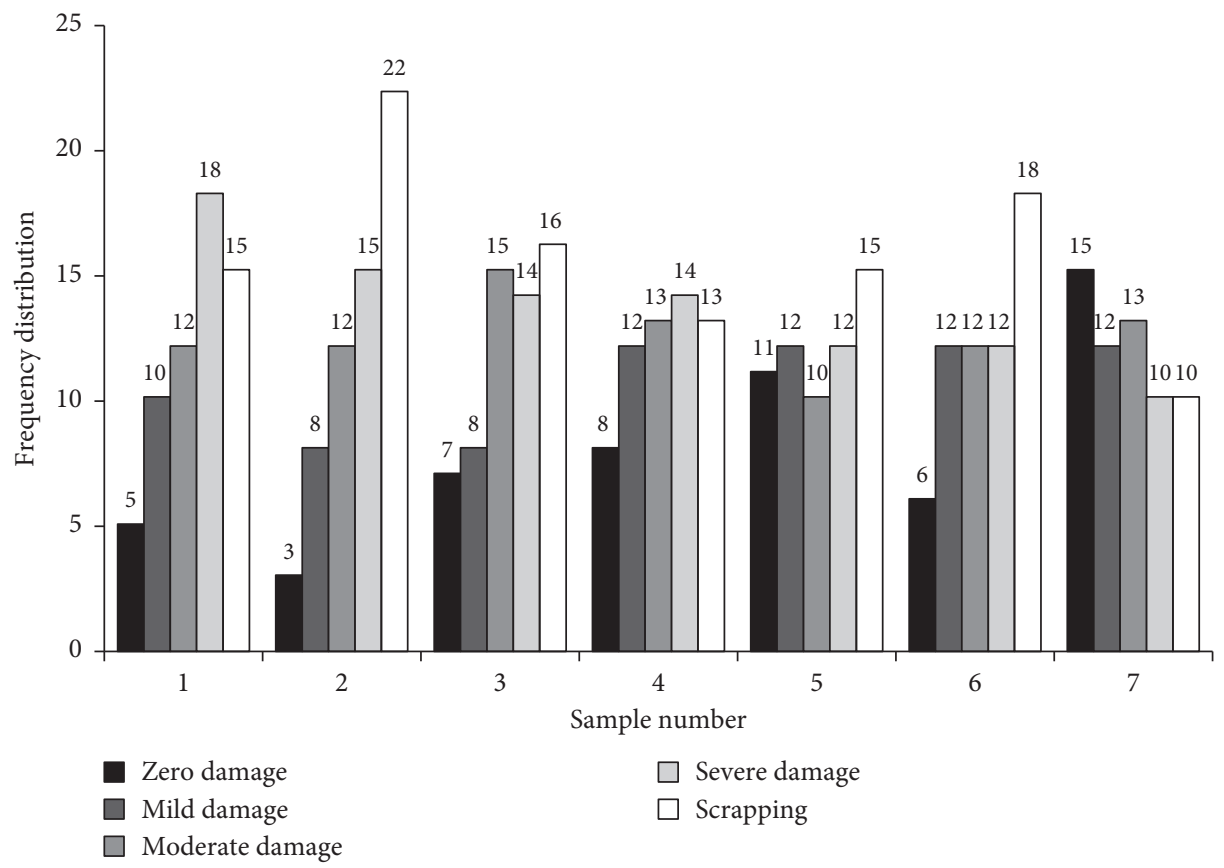

FIGURE 1: Frequency distribution of targets with different damage grades.

of iterations of each chain to 1500 and updating it in every 100. The convergence of Bayesian inference results based on MCMC needs to be judged, and Figures 2 and 3 show the iterative trace diagram and autocorrelation function diagram of parameters $\theta_{1}, \theta_{2}, \theta_{3}, \theta_{4}, \theta_{5}$, and according to the diagrams, whether the Markov chain converges or not can be decided.

We found all three Markov chains converge by observing the iterative trace graph and autocorrelation function graph. Then, the posterior estimation of parameters can be performed, which is shown in Figure 4.

After 1500 iterations of each chain, the posterior estimation results of parameters $\theta_{1}, \theta_{2}, \theta_{3}, \theta_{4}, \theta_{5}$ are shown in Table 3, including the estimated mean value of each parameter, MCMC iteration error, and $97.5 \%$ confidence interval of the setting.

In the actual attack, the damage grade of the target cannot be determined. Assuming that the threshold value $\Lambda$ of damage probability is 0.8 , and then the cumulative value of ammunition required for different damage grades can be obtained; that is, the optimal ammunition demand $\varphi^{\Gamma_{r}^{*}}$ for reaching each damage grade can be obtained by referring to equation (19). The grade of zero damage means that the target equipment's combat performance is nearly unaffected, and no ammunition is consumed. Table 4 shows the optimal ammunition demand for four damage grades: mild damage, moderate damage, severe damage, and scrapping.

From the calculation results in Table 4, it can be seen that the ammunition consumption is $1,2,4$, and 8 rounds, respectively, corresponding with the four damage levels: mild damage, moderate damage, severe damage, and scrapping. Therefore, with the condition of small samples, the ammunition demand for reaching different damage levels based on the Bayesian inference method is obtained, which is economical and scientific. Considering the prior information, the Bayesian method can overcome the problem of small sample size and has its superiority in prediction. 

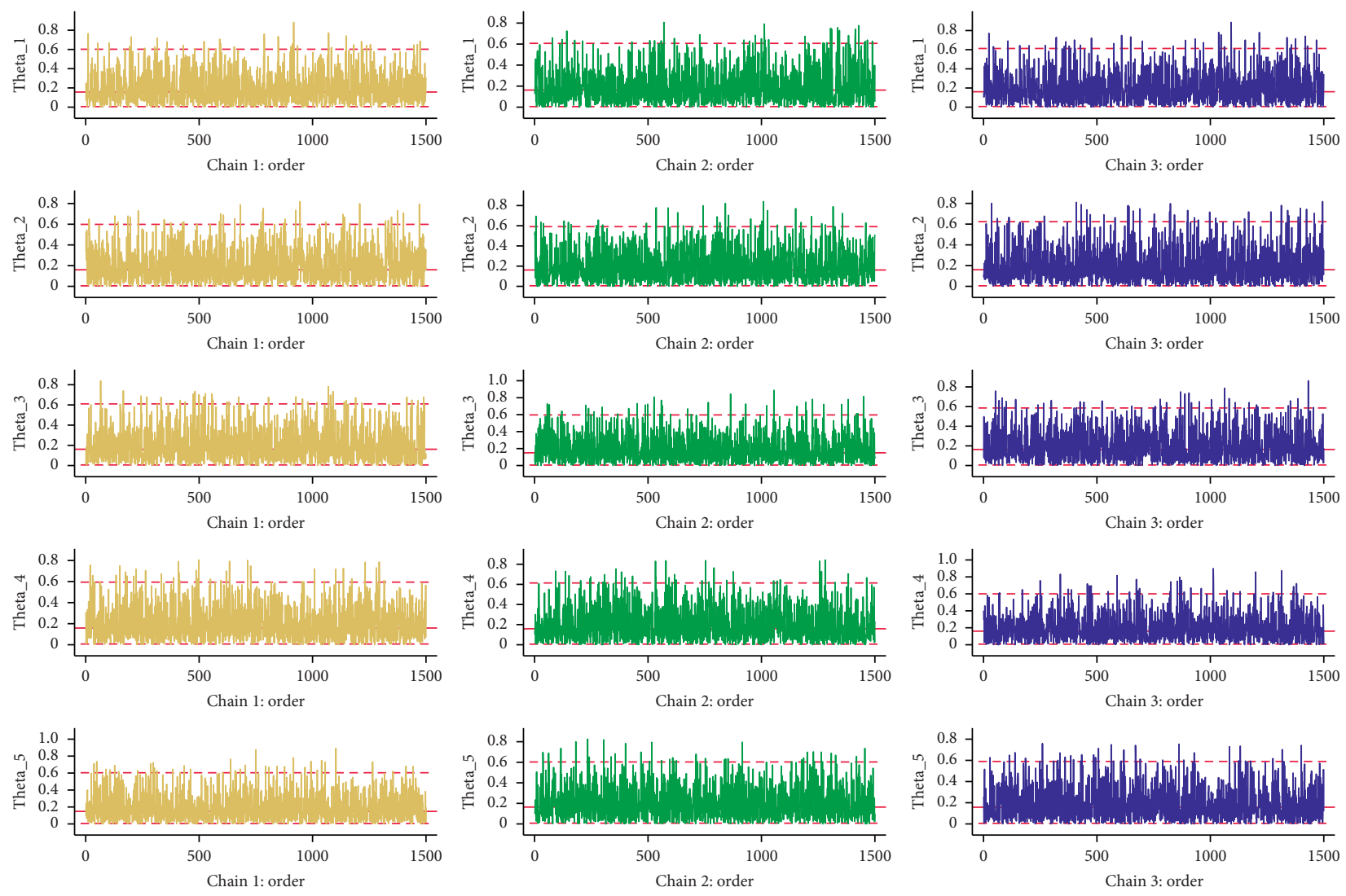

FIGURE 2: Iterative tracer diagram for parameters $\theta_{1}, \theta_{2}, \theta_{3}, \theta_{4}, \theta_{5}$.

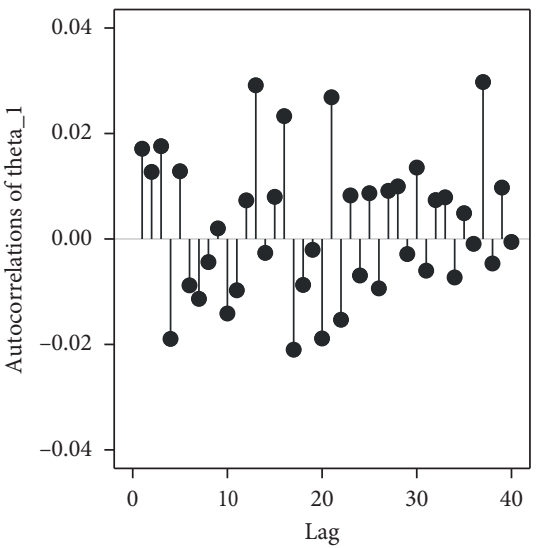

(a)

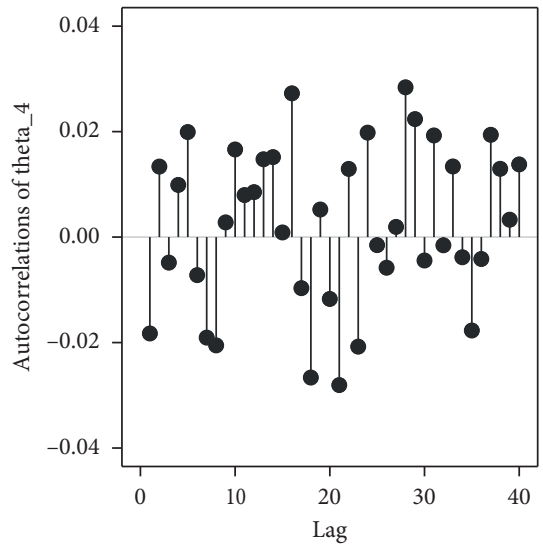

(d)

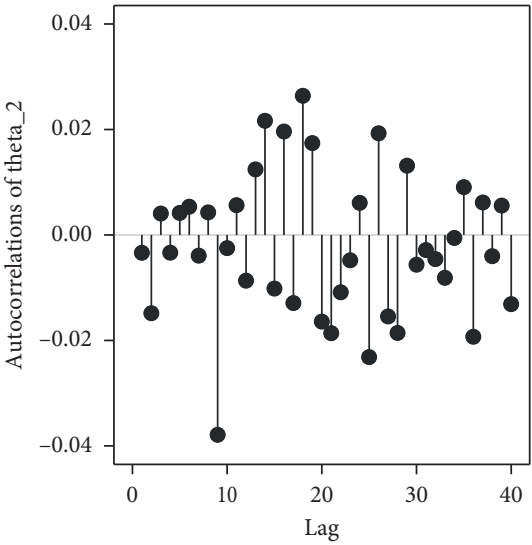

(b)

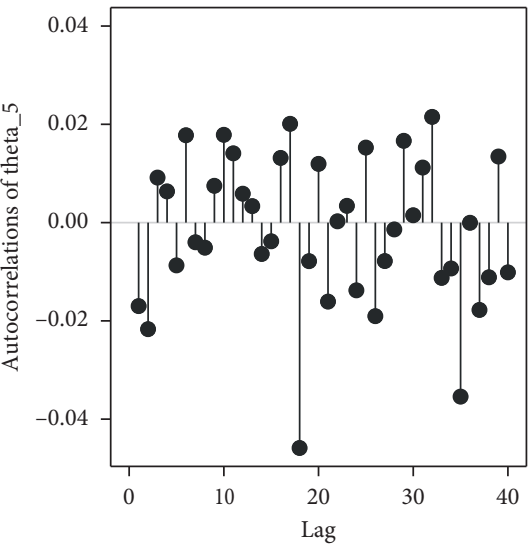

(e)

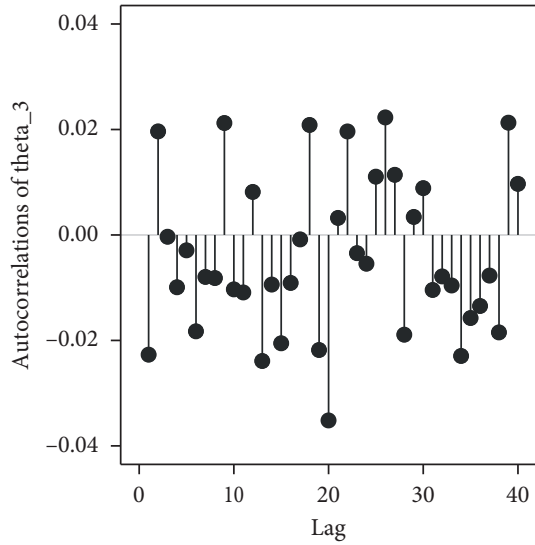

(c)

FIgURE 3: Autocorrelation function diagram of parameters $\theta_{1}, \theta_{2}, \theta_{3}, \theta_{4}, \theta_{5}$. 


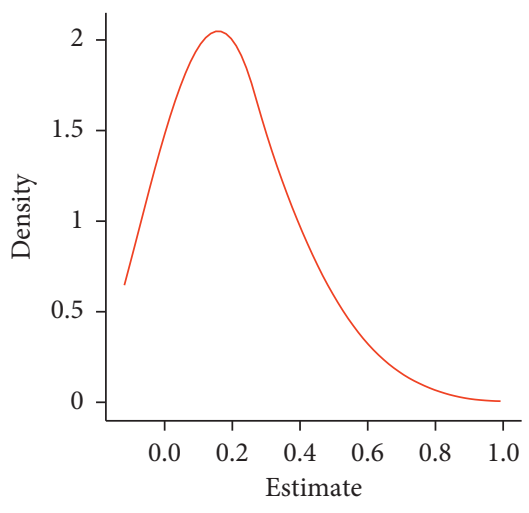

(a)

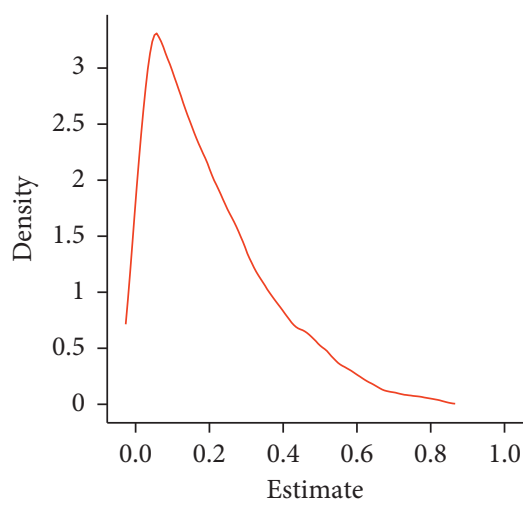

(b)

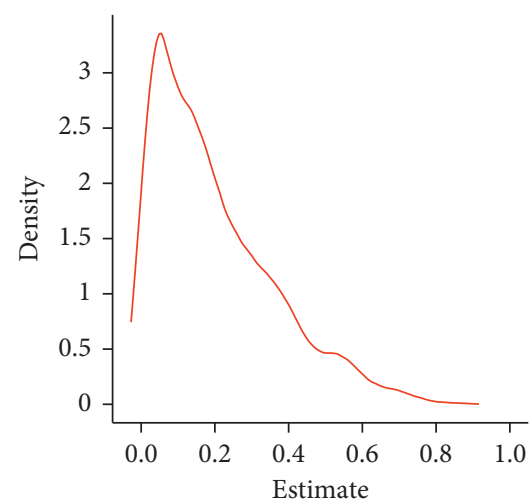

(c)

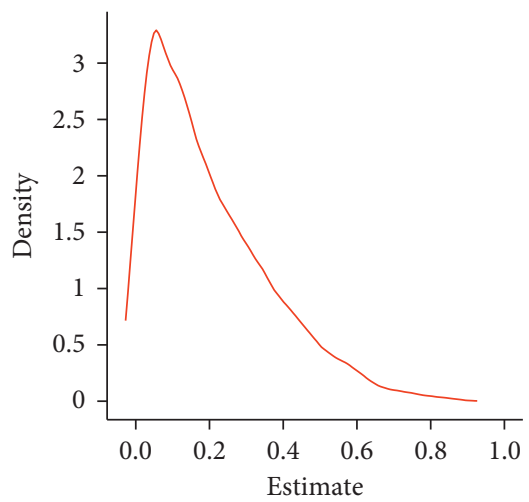

(d)

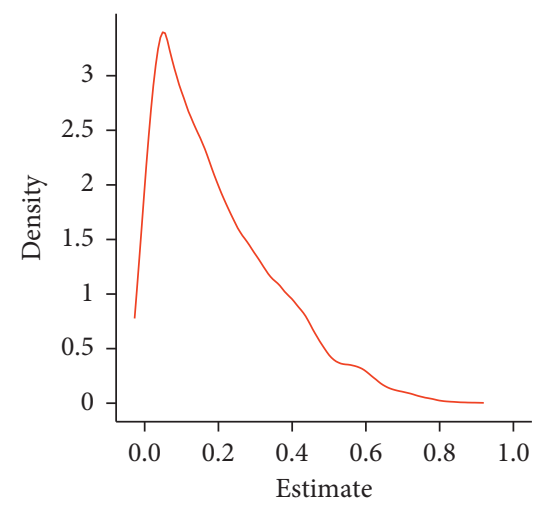

(e)

Figure 4: Nuclear density diagram of parameters $\theta_{1}, \theta_{2}, \theta_{3}, \theta_{4}, \theta_{5}$. (a) theta_1, (b) theta_2, (c) theta_3, (d) theta_4, and (e) theta_5.

TABle 3: Posterior statistics of parameters $\theta_{1}, \theta_{2}, \theta_{3}, \theta_{4}, \theta_{5}$.

\begin{tabular}{lcccccr}
\hline Parameter & $n$ & Mean & SD & MC error & Median & $97.5 \%$ CrI \\
\hline theta_1 & 4500 & 0.1997 & 0.1632 & 0.0026 & 0.1603 & $(0.006,0.609)$ \\
theta_2 & 4500 & 0.2009 & 0.1635 & 0.0023 & 0.1607 & $(0.005,0.608)$ \\
theta_3 & 4500 & 0.1991 & 0.1637 & 0.0023 & 0.1591 & $(0.006,0.600)$ \\
theta_4 & 4500 & 0.2017 & 0.1645 & 0.0025 & 0.1591 & $(0.007,0.601)$ \\
theta_5 & 4500 & 0.1986 & 0.1631 & 0.0024 & 0.1575 & $(0.006,0.595)$ \\
\hline
\end{tabular}

TABle 4: Optimal ammunition demand for each damage grade.

\begin{tabular}{|c|c|c|c|}
\hline Damage grade & Ammunition amount & Cumulative destruction injury probability & Whether optimal \\
\hline Mild damage & 1 & 0.8003 & $\sqrt{ }$ \\
\hline Mild damage & 2 & 0.9601 & \\
\hline Moderate damage & 1 & 0.5994 & \\
\hline Moderate damage & 2 & 0.8395 & $\sqrt{ }$ \\
\hline Moderate damage & 3 & 0.9357 & \\
\hline Severe damage & 1 & 0.4003 & \\
\hline Severe damage & 2 & 0.6404 & \\
\hline Severe damage & 3 & 0.7843 & \\
\hline Severe damage & 4 & 0.8707 & $\sqrt{ }$ \\
\hline Severe damage & 5 & 0.9224 & \\
\hline Scrapping & 1 & 0.1986 & \\
\hline Scrapping & 2 & 0.3578 & \\
\hline Scrapping & 3 & 0.4853 & \\
\hline Scrapping & 4 & 0.5875 & \\
\hline Scrapping & 5 & 0.6694 & \\
\hline Scrapping & 6 & 0.7351 & \\
\hline Scrapping & 7 & 0.7877 & \\
\hline Scrapping & 8 & 0.8299 & $\sqrt{ }$ \\
\hline
\end{tabular}




\section{Conclusions}

To predict the ammunition demand for reaching different damage grades, the Bayesian inference method based on multinomial distribution is proposed by fully considering the current situation of little data in ammunition strike trials, which provides a reference for the prediction of ammunition demand under the condition of small samples in the trial of high-tech weapons and ammunition. The main work accomplished includes the following:

(1) To provide a prerequisite for Bayesian inference, multinomial distribution assumptions are made in the process of ammunition hitting.

(2) With the fusion of multisource prior information based on the D-S theory, multiple information sources can be taken full advantage of. This paper adopts the classical fusion rule, but when there is a large amount of data, there may be conflicts between evidence, so this rule will no longer be applicable. Therefore, a new basic probability assignment method proposed by Jing et al. can be considered to adopt [33] and a new classification method based on the negation of a basic probability assignment in the evidence theory proposed by $\mathrm{Wu}$ et al. [34] to resolve possible conflict information fusion in the method.

(3) On the basis of analyzing the prior information, this paper chooses the Dirichlet distribution, which is the conjugate distribution of multinomial distributions, as the prior distribution of the Bayesian inference model of ammunition demand, that not only does it simplify the calculation but also the posterior distribution parameters are better explained.

(4) By using the MCMC method based on Gibbs sampling to solve the Bayesian inference model of ammunition demand based on multinomial distribution and with the application of WinBUGS software to simulation, the ammunition demand for different damage grades is obtained with the idea that the cumulative probability exceeds the given probability value.

To sum up, the ammunition demand forecasting method in this paper makes the demand forecasting of a new type of ammunition feasible, which has practical guidance to the ammunition supply support, yet it is necessary to consider the damage efficiency of new ammunition in further study.

\section{Data Availability}

All the data supporting this study are included within this paper.

\section{Conflicts of Interest}

The authors declare that they have no conflicts of interest.

\section{Acknowledgments}

Our work was supported by army scientific research (LJ20202C050369).

\section{References}

[1] X. Shi, R. Zhao, X. Luo, J. Zhang, and X. Song, "Research on ammunition conversion coefficient of plateau cold region based on fuzzy BP neural network," Fire Control Command Control, vol. 45, no. 2, pp. 59-63, 2020.

[2] R. Zhao, X. Shi, Q. Wang, X. Su, and X. Song, "Bayesian inference for ammunition demand based on Gompertz distribution," Journal of Systems Engineering and Electronics, vol. 31, no. 3, pp. 567-577, 2020.

[3] H. Xue, Y. Wang, T. Zhang, and T. Liu, "Demand forecasting model for joint fire strike ammunition under stochastic combination constraints," Acta Armamentarii, vol. 40, no. 8, pp. 1716-1724, 2019.

[4] Q. Zou, C. Wang, R. Jia, and D. Wang, "Research on prediction methods of wartime ammunition consumption," Journal of Ordnance Equipment Engineering, vol. 38, no. 9, pp. 12-16, 2017.

[5] X. Xu, Y. Tan, and H. Xu, "On prediction methods of wartime ammunition consumption," Journal of Systems Simulation, vol. 25, no. S1, pp. 358-361, 2013.

[6] Y. Xie, M. Xiao, L. Zhao, and P. Yao, "Research on air-to-air missile consumption demand forecasting model," Computers in Simulation, vol. 33, no. 9, pp. 90-96, 2016.

[7] D. Tian and J. He, "Aviation ammunition consumption prediction model based on mutated particle swarm optimization and deep neural network," Journal of Nanjing University of Science and Technology, vol. 42, no. 6, pp. 716-721+726, 2018.

[8] P. Zhu and W. Zhao, "Application of combination forecasting method in ammunition consumption forecasting," Science Technology Engineering, vol. 7, no. 14, pp. 3561-3563, 2007.

[9] M. Beer, S. Ferson, and V. Kreinovich, "Imprecise probabilities in engineering analyses," Mechanical Systems and Signal Processing, vol. 37, no. 1-2, pp. 4-29, 2013.

[10] J. Zhang, "Uncertainty quantification from small data: a multimodel approach," Dissertation thesis, Johns Hopkins University, Baltimore, MD, USA, 2018.

[11] J. Zhang and M. D. Shields, "On the quantification and efficient propagation of imprecise probabilities resulting from small datasets," Mechanical Systems and Signal Processing, vol. 98, pp. 465-483, 2018.

[12] M. Faes, M. Broggi, E. Patelli et al., "Inverse quantification of epistemic uncertainty under scarce data: Bayesian or interval approach?" in Proceedings of the 13th International Conference on Applications of Statistics and Probability in Civil Engineering, Seoul, South Korea, May 2019.

[13] M. Faes, M. Broggi, E. Patelli et al., "A multivariate interval approach for inverse uncertainty quantification with limited experimental data," Mechanical Systems and Signal Processing, vol. 118, pp. 534-548, 2019.

[14] D. Wu and Y. Tang, "An improved failure mode and effects analysis method based on uncertainty measure in the evidence theory," Quality and Reliability Engineering International, vol. 36, no. 5, pp. 1786-1807, 2020.

[15] Y. Tang, D. Wu, and Z. Liu, "A new approach for generation of generalized basic probability assignment in the evidence theory," Pattern Analysis and Applications, pp. 1-17, 2021. 
[16] J. Zhang and Y. Deng, "A method to determine basic probability assignment in the open world and its application in data fusion and classification," Applied Intelligence, vol. 46, no. 4, pp. 934-951, 2017.

[17] D. Gan, B. Yang, and Y. Tang, "An extended base belief function in Dempster-Shafer evidence theory and its application in conflict data fusion," Mathematics, vol. 8 , no. 12 , 2020.

[18] Z. Gu, A method of small sample reliability assessment based on Bayesian theory, 2017.

[19] J. Zhang and M. D. Shields, "The effect of prior probabilities on quantification and propagation of imprecise probabilities resulting from small datasets," Computer Methods in Applied Mechanics and Engineering, vol. 334, pp. 483-506, 2018.

[20] E. Shitsi, E. K. Boafo, F. Ameyaw, and H. C. Odoi, "Investigating the effect of prior distributions on posterior estimates of common cause failure parameters using Bayesian method," Journal of Nuclear Engineering and Radiation Science, vol. 6, no. 3, 2020.

[21] S. Watanabe, Mathematical Foundations of Bayesian Statistics, Chapman \& Hall/CRC, Boca Raton, FL, USA, 2018.

[22] X. Zhang, Researches on the small sample statistical inference and fusion theory and its application to the assessment of weapon system, 2003.

[23] Z. Chen and Y. Liu, "Test method of prior information based on Bayes reliability evaluation," Journal of Electronic Measurement and Instrument, vol. 22, no. S2, pp. 41-44, 2008.

[24] C. Wang, J. Qiu, G. Liu, and Y. Zhang, "Testability evaluation using prior information of multiple sources," Chinese Journal of Aeronautics, vol. 27, no. 4, pp. 867-874, 2014.

[25] X. Zhu, "Review of Markov Chain Monte Carlo method," Statistics \& Decisions, vol. 21, pp. 151-153, 2009.

[26] F. Ye, Markov Chain Monte Carlo method and implemented with R, 2014.

[27] Z. Xiao and J. Zhu, "Neural sampling," Chinese Journal of Computers, vol. 40, no. 6, pp. 1379-1393, 2017.

[28] C. Cao, The parameter estimation of the statistics models based on the MCMC method, 2007.

[29] E. I. George and R. E. McCulloch, "Variable selection via Gibbs sampling," Journal of the American Statistical Association, vol. 88, no. 423, pp. 881-889, 1993.

[30] A. E. Gelfand, "Gibbs sampling," Journal of the American Statistical Association, vol. 95, no. 452, pp. 1300-1304, 2000.

[31] K. K. Osmundsen, T. S. Kleppe, and A. Oglend, "MCMC for Markov-switching models-Gibbs sampling vs. marginalized likelihood," Communications in Statistics-Simulation and Computation, vol. 50, no. 2, 2019.

[32] L. Jing and Z. Huiming, "Study on storage reliability evaluation for ammunition using Gibbs sampler," Journal of China Ordnance, vol. 4, pp. 268-271, 2007.

[33] M. Jing and Y. Tang, "A new base basic probability assignment approach for conflict data fusion in the evidence theory," Applied Intelligence, vol. 51, no. 2, pp. 1056-1068, 2021.

[34] D. Wu, Z. Liu, and Y. Tang, "A new classification method based on the negation of a basic probability assignment in the evidence theory," Engineering Applications of Artificial Intelligence, vol. 96, p. 103985, 2020. 